\title{
Medial clavicle fracture with bone destruction after radical neck dissection combined with postoperative chemotherapy for secondary cervical lymph node metastasis of tongue cancer: a case report
}

\author{
Masayasu Tashiro $^{1} \cdot$ Tomoaki Sano $^{1} \cdot$ Kazutaka Sugiura $^{1} \cdot$ Yasuhito Minamida $^{2} \cdot$ Yoshihiro Abiko $^{3} \cdot$ Hiroki Nagayasu $^{2}$. \\ Eiji Nakayama ${ }^{1}$
}

Received: 13 December 2020 / Accepted: 21 January 2021 / Published online: 12 February 2021

(c) The Author(s) 2021

\begin{abstract}
Background Clavicle fractures (CF) after radical neck dissection (RND) for oral cancer are rare but are thought to occur as a result of myotonia and decreased blood supply to the muscles around the clavicle after RND. The current report presents a rare case of a non-neoplastic pathological CF after RND, and discusses the role of imaging examinations for the timely detection of CF.

Case report An 82-year-old Japanese man underwent RND followed by chemotherapy without radiotherapy for secondary metastasis of the right cervical lymph node after resection of tongue cancer. Computed tomography at 6 months after RND revealed a fracture with bone destruction in the proximal end of the right clavicle. He had no history of trauma at the site of the fracture and no symptoms. The possibility of bone metastasis of the clavicle was considered; however, the bone destruction had not advanced 6 years after the discovery of the fracture. The CF was thus finally considered to be a side effect of RND, rather than metastasis.

Conclusion CF is a rare complication following treatment for head and neck cancer but can be caused by neck dissection. Regular imaging examinations, including the clavicular region, are therefore needed before and after surgery to ensure the timely detection of CF.
\end{abstract}

Keywords Clavicle fracture $\cdot$ Oral cancer $\cdot$ Radical neck dissection · Diagnostic imaging

Eiji Nakayama

enaka@hoku-iryo-u.ac.jp

1 Division of Oral and Maxillofacial Radiology, Department of Human Biology and Pathophysiology, School of Dentistry, Health Sciences University of Hokkaido, 1757 Kanazawa, Ishikari-Tobetsu, Hokkaido 061-0293, Japan

2 Division of Oral Maxillofacial Surgery, Department of Human Biology and Pathophysiology, School of Dentistry, Health Sciences University of Hokkaido, 11757 Kanazawa, Ishikari-Tobetsu, Hokkaido, 061-0293, Japan

3 Division of Oral Medicine and Pathology Department of Human Biology and Pathophysiology School of Dentistry, Health Sciences University of Hokkaido, 1757 Kanazawa, Ishikari-Tobetsu, Hokkaido, 061-0293, Japan

\section{Introduction}

Traumatic clavicle fractures $(\mathrm{CF})$ are relatively common, accounting for $4 \%$ of adult fractures [1]. CF are generally classified as medial- or middle-third fractures, or fractures distal to the coracoclavicular ligament, according to Allman's classification [2, 3], with relative incidences of approximately $2.8 \%, 69.2 \%$, and $28 \%$, respectively [3].

In contrast, non-traumatic fractures in patients with malignant tumors are usually caused by bone metastases, but may also rarely occur after radical neck dissection (RND). Notably, fractures of the medial end of the clavicle have been reported as a rare late complication after RND, with an incidence of approximately $0.4 \%-0.5 \%$ [4]. Regarding their cause, Strauss et al. reported that RND or radiotherapy caused weakening of the bone and blood supply, resulting in subsequent fracture of the clavicle [5].

We recently experienced a case of a non-neoplastic fracture in the medial third of the clavicle with bone destruction 
after RND combined with postoperative chemotherapy, with no radiotherapy. We, therefore, present this rare case of a non-neoplastic CF after RND and chemotherapy, and discuss the role of imaging examinations for the timely detection of CF.

\section{Case report}

An 81-year-old Japanese man visited our hospital with a chief complaint of pain in the right lingual margin. The patient had been aware of a mass on the right lingual surface for 4 months (Fig. 1), and finally attended our hospital because of contact pain with his dentures.

Contrast-enhanced computed tomography (CT) showed an enhanced soft tissue mass at the right lingual margin (Fig. 2), but no lymphadenopathies suggesting metastasis. Examination of a biopsy of the lingual mass revealed squamous cell carcinoma, with clinical TNM classification T1N0M0 (stage I). The patient was therefore hospitalized and underwent tumor resection of the right lingual site under general anesthesia on June 25, 2009.

Follow-up contrast-enhanced CT 8 months after tumor resection (February 15, 2010) showed a lymph node swelling at the right upper cervical region, suggesting secondary lymph node metastasis (Fig. 3). He, therefore, underwent RND under general anesthesia on March 19, 2010, according to the traditional method [6]. Postoperative adjuvant chemotherapy with fluorouracil (total dose: $8750 \mathrm{mg}$ ), cisplatin (total dose: $240 \mathrm{mg}$ ), and tegafur/gimeracil/oteracil (total dose: $1680 \mathrm{mg}$ ) was planned. However, because of the patient's poor physical condition, adjuvant chemotherapy was stopped after administration of $8750 \mathrm{mg}$ fluorouracil, $240 \mathrm{mg}$ cisplatin, and $1560 \mathrm{mg}$ of tegafur/gimeracil/oteracil.

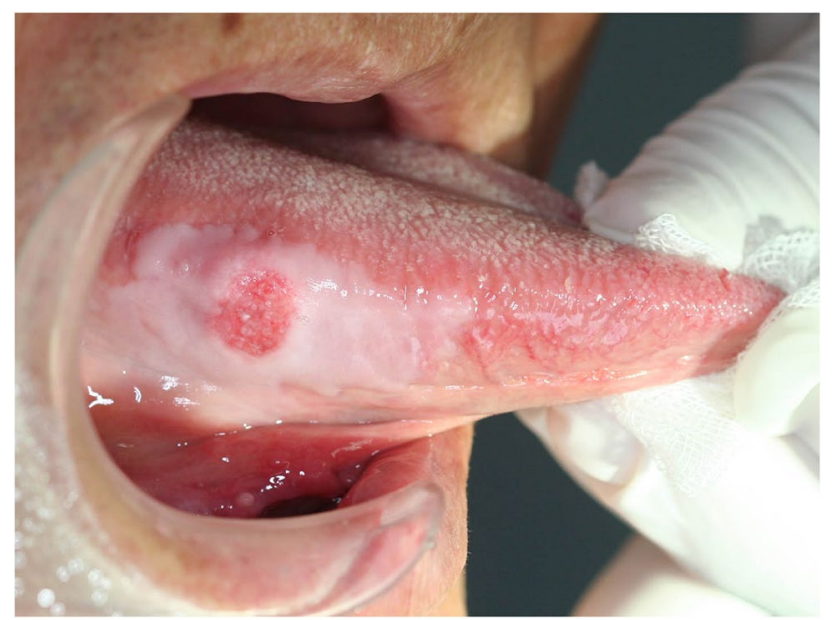

Fig. 1 Tumor with large leukoplakia observed in the right margin of the tongue

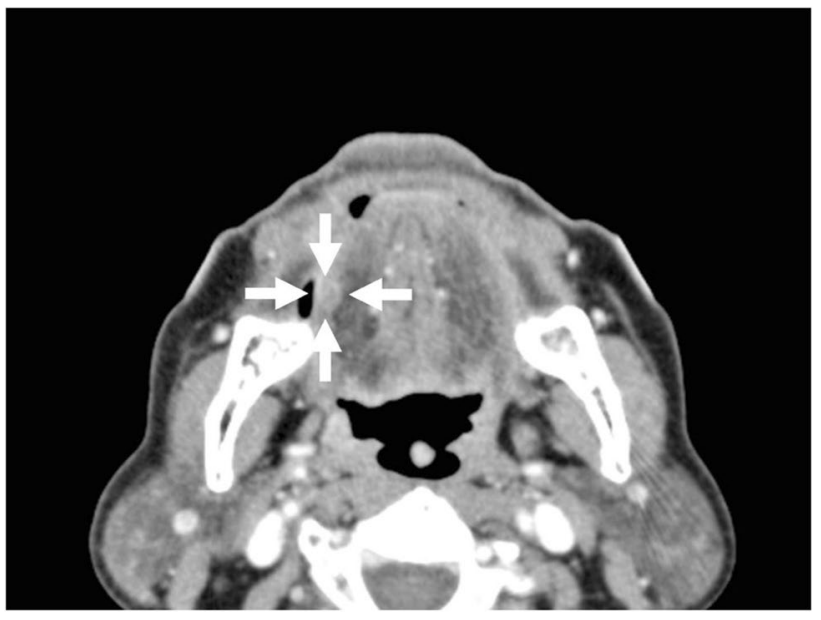

Fig. 2 Contrast-enhanced CT revealed an enhanced soft tissue mass (arrows) at the right lingual margin, diagnosed histopathologically as squamous cell carcinoma

Follow-up contrast-enhanced CT 6 months after right RND (September 17, 2010) detected a fracture with bone destruction in the proximal end of the right clavicle (Fig. 4). This was regarded as a pathologic fracture, and bone metastasis from the tongue cancer was considered as a possible cause.

The patient underwent ${ }^{18} \mathrm{~F}$-fluorodeoxyglcose (FDG) positron emission tomography (PET)-CT on September 24, 2010 , which showed moderate and relatively diffuse ${ }^{18} \mathrm{~F}$ FDG accumulation at the proximal end of the right clavicle, corresponding to the fracture site (Fig. 5). However, the level of accumulation was not very high and the maximum

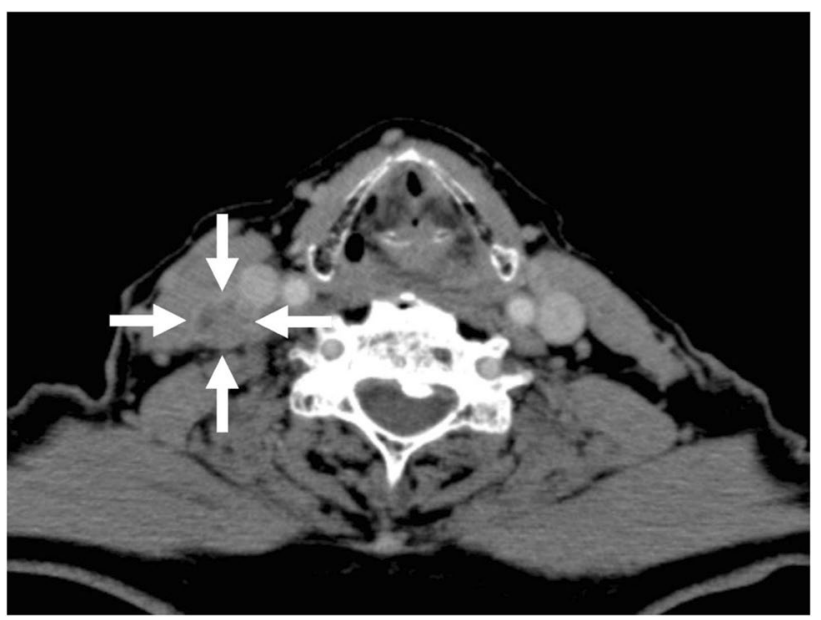

Fig. 3 Contrast-enhanced CT 6 months after tumor resection showed a new swelling (arrows) of the right upper cervical lymph node, suggesting metastasis 
Fig. 4 Contrast-enhanced CT (a: bone-window image, $\mathbf{b}$ : soft tissue-window image) on September 17, 2010, 6 months after right RND, showed a fracture with bone destruction (arrows) at the proximal end of the right clavicle
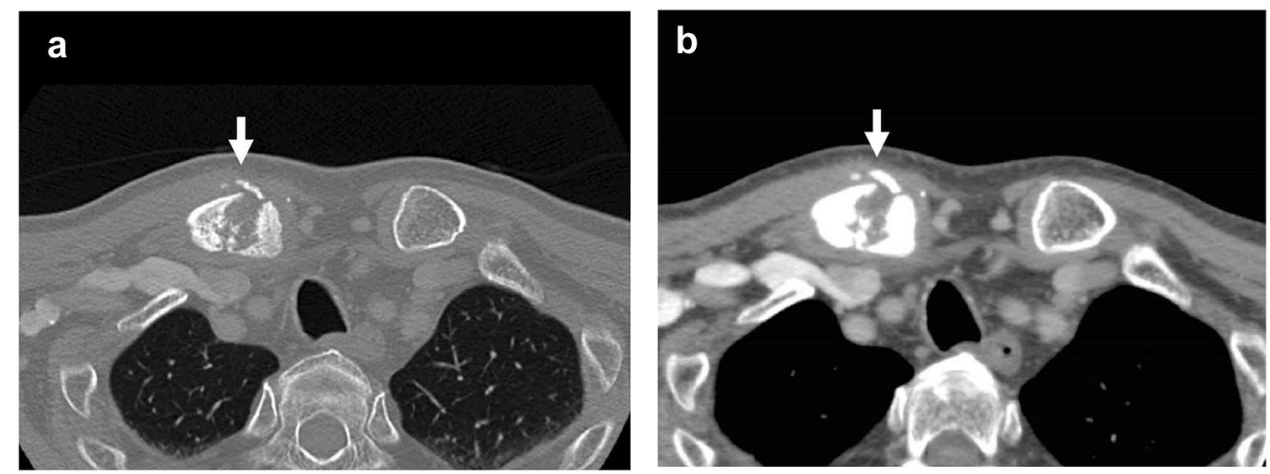

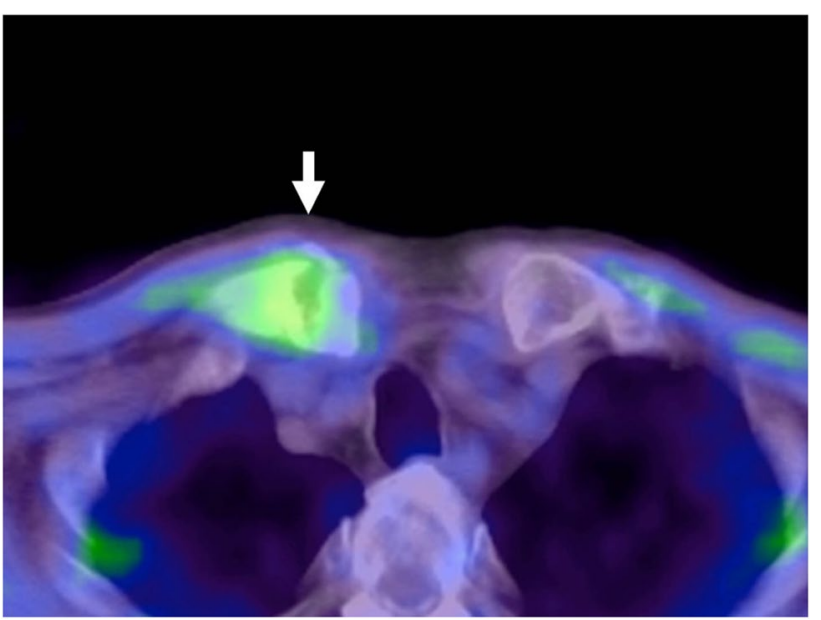

Fig. 5 PET-CT on September 24, 2010, 1 week after detection of CF by $\mathrm{CT}$, showed relatively weak accumulation (arrow) at the fractured clavicle

standardized uptake value was 3.7 , and the probability of metastasis was therefore thought to be low.

Consultation 4 days after PET-CT detected swelling and slight redness of the superficial skin on the right clavicle. However, the patient reported no symptoms in the right clavicular region, and no history of trauma that might have caused a fracture of the clavicle.

Ultrasonography (US) on October 5, 2010 showed a hypoechoic solid mass destroying the cortex of the clavicle, with weak signals in the internal mass and surrounding blood flow on power Doppler US (Fig. 6), suggesting that the $\mathrm{CF}$ was not caused by metastasis. Moreover, the redness disappeared 1 week after it was first noticed, although the swelling remained.

We, therefore, considered that the $\mathrm{CF}$ was likely to have been caused by non-neoplastic changes, rather than by bone metastasis, and accordingly adopted a watch and wait approach regarding the $\mathrm{CF}$.

Four follow-up contrast-enhanced CT scans were carried out over the following 6 years, with no signs of recurrence

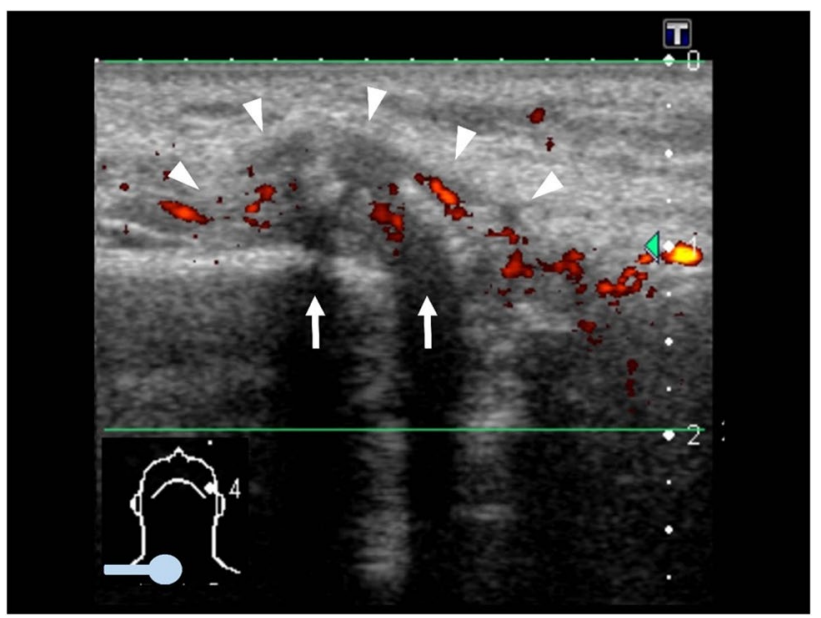

Fig. 6 Power Doppler US on October 5, 2010 showed a hypoechoic solid mass (arrowheads) destroying the cortex of the clavicle (arrows), and with weak signals in the internal mass and surrounding blood flow

or metastasis of the malignant tumor. Regarding the CF, the separation between the bone fragments reduced and the patient's condition was stable without inflammation, although adhesion of the bone fragments was not achieved (Fig. 7).

In conclusion, we judged that the $\mathrm{CF}$ had occurred as a secondary effect of RND.

\section{Discussion}

We experienced a patient with a medial CF following RND in 2010. We performed a total of 25 RND procedures from 2008 to 2019, and the present patient represented the only case of a CF after RND.

However, similar cases of $\mathrm{CF}$ after neck dissection have been reported by other researchers [4, 5, 7-15], who advocated various hypotheses regarding their causes.

Strauss et al. [5] and others [4, 7, 8, 14] considered that $\mathrm{CF}$ after neck dissection was a result of weakening of the 

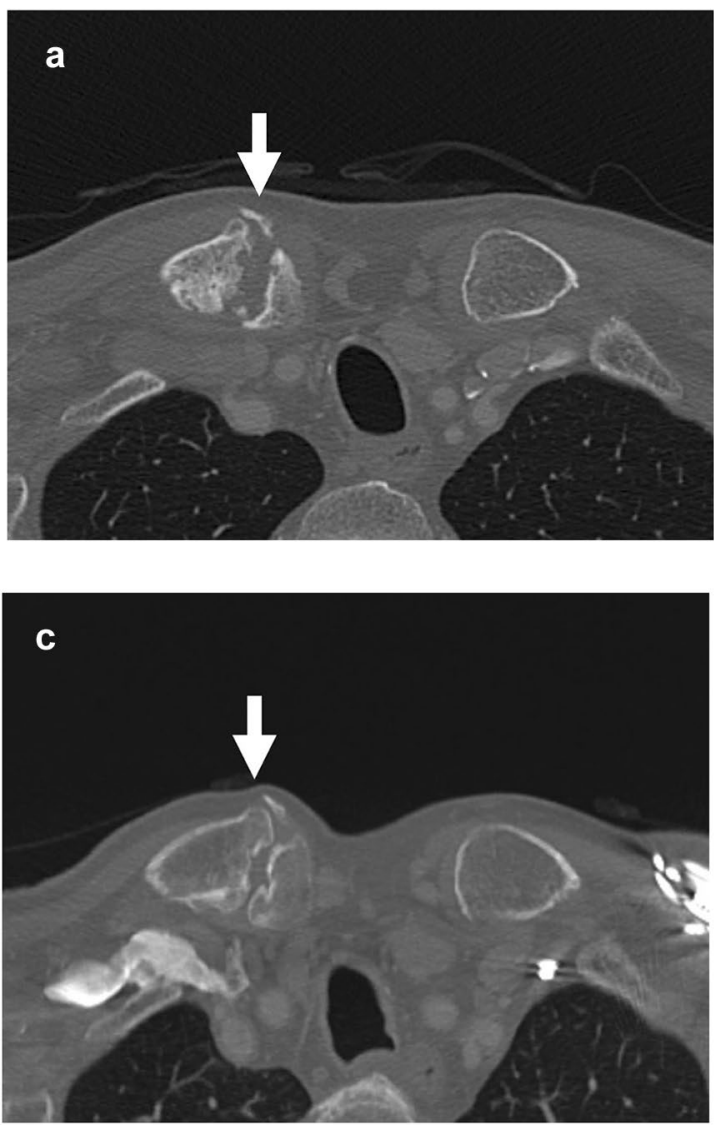

Fig. 7 Follow-up contrast-enhanced CT examinations over the next 6 years showed gradual narrowing of the separation between the bone fragments, but no adhesion of the fragments (arrows). a: CT on June

bone and blood supply caused by neck dissection and/or radiotherapy. However, the current patient did not receive radiation therapy. Other reports of medial $\mathrm{CF}$ after neck dissection indicated that neck dissection alone might cause such fractures, with or without postoperative radiotherapy $[11,14]$.

Regarding the possible association between CF after neck dissection and bone destruction, Shodo et al. [13] reported a case of $\mathrm{CF}$ after neck dissection and postoperative radiotherapy followed by osteomyelitis with bone destruction. They suggested that osteomyelitis and abscess formation with bone destruction caused by risk factors such as radiotherapy, tracheostomy, and adjacent infection might complicate the fracture findings, such as bone destruction in clavicle stress fractures after neck dissection [13]. In both the current and Shodo et al.'s case, the fracture was accompanied by bone destruction; however, our case was not associated with infection.

Fujimoto et al.'s [14] study was also relevant to the association between $\mathrm{CF}$ after neck dissection and bone destruction. They assessed the CT images of nine cases of $\mathrm{CF}$ following neck dissection and suggested that proximal
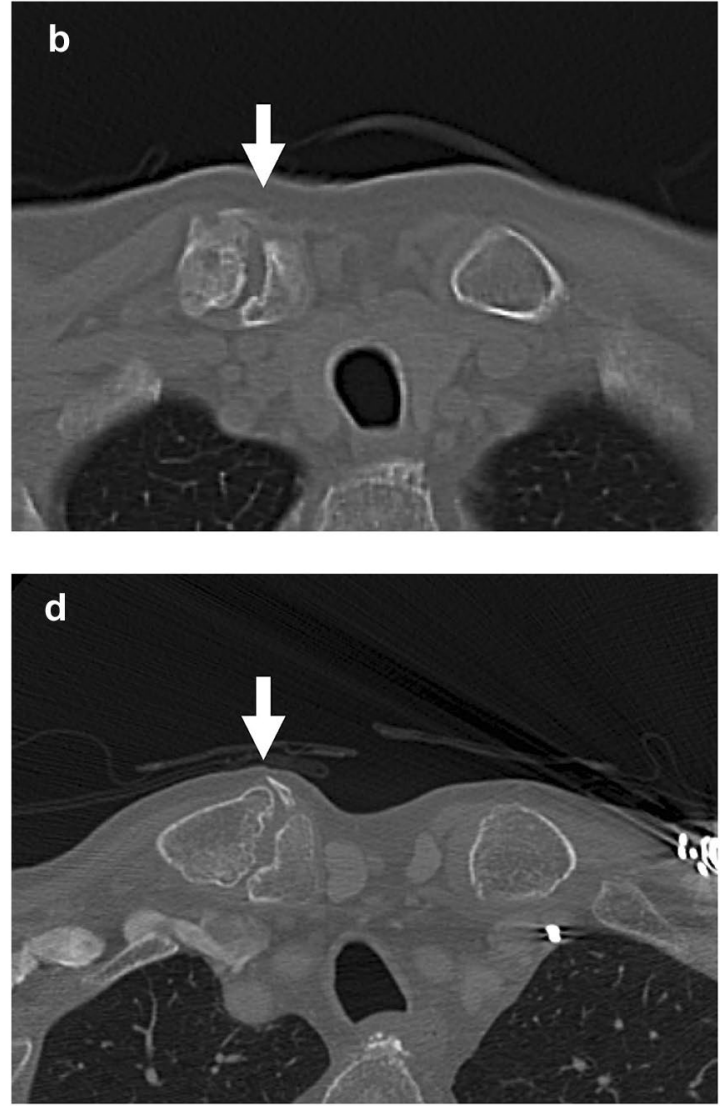

20, 2011; b: CT on May 20, 2013, c: CT on November 8, 2016, d: CT on November 2, 2017

CF consistently showed an extraosseous soft-tissue mass formation without bone destruction on CT [14]. In our case, the patient had no subjective symptoms related to the fracture, despite the formation of an extraosseous soft-tissue mass, as in Fujimoto et al.'s cases. However, the fracture in the present case was accompanied by bone destruction, which was absent in Fujimoto et al.'s cases.

The pathogenesis of bone destruction in CF after neck dissection might be associated with multiple factors, and the cause for the bone destruction in the current patient has not yet been identified. However, comparing the present and previous cases of $\mathrm{CF}$, our case suggests that postoperative chemotherapy after RND might contribute to bone destruction in $\mathrm{CF}$, even in the absence of radiotherapy [8, 16] and infection [14] of the clavicle. The influence of chemotherapy on the occurrence of $\mathrm{CF}$ after neck dissection remains unknown. One study reported a possible effect of chemotherapy on bone [17], while some cases of $\mathrm{CF}$ following neck dissection have been reported in patients without chemotherapy [7-10], and others have been noted in cases with chemotherapy [8,9]. Further studies are therefore needed to elucidate the influence 
of chemotherapy in the pathogenesis of CF after neck dissection.

In the present patient, the $\mathrm{CF}$ was first discovered during a follow-up imaging examination, and the $\mathrm{CF}$ status during the 6 months from RND to its first discovery remains unknown. We are therefore unable to explain the interaction between the fracture and the bone destruction. Future cases should thus consider the mechanism of bone destruction in CF after neck dissection.

The current case shows that CF after RND may be accompanied by bone destruction, even if the fracture is not associated with infection. Moreover, in our case, bone destruction of the CF might have progressed because the discovery of the fracture was delayed. This case highlights the need to recognize $\mathrm{CF}$ as one of the adverse events after neck dissection [14], and to ensure that the condition is promptly detected and treated. Regular follow-up imaging examinations, including CT, will aid the rapid discovery of CF after neck dissection for head and neck cancer [8, 13, 14].

$\mathrm{CF}$ is a rare complication after treatment for head and neck cancer but can be caused by neck dissection. Regular imaging examinations, including the clavicular region, should, therefore, be carried out before and after surgery to ensure the prompt detection of CF.

Acknowledgements We thank Susan Furness, PhD, from Edanz Group (https://en-author-services.edanzgroup.com/ac) for editing a draft of this manuscript.

Funding Not applicable.

\section{Compliance with ethical standards}

Conflicts of interest There are no financial or other relations that could lead to a conflict of interest.

Ethical standard This report was exempt from ethical approval by our institution because all identifiable patient information has been removed.

Informed consent Written informed consent was obtained from the patient to use this case for the purposes of education and study.

Open Access This article is licensed under a Creative Commons Attribution 4.0 International License, which permits use, sharing, adaptation, distribution and reproduction in any medium or format, as long as you give appropriate credit to the original author(s) and the source, provide a link to the Creative Commons licence, and indicate if changes were made. The images or other third party material in this article are included in the article's Creative Commons licence, unless indicated otherwise in a credit line to the material. If material is not included in the article's Creative Commons licence and your intended use is not permitted by statutory regulation or exceeds the permitted use, you will need to obtain permission directly from the copyright holder. To view a copy of this licence, visit http://creativecommons.org/licenses/by/4.0/.

\section{References}

1. Nordqvist A, Petersson C. The incidence of fractures of the clavicle. Clin Orthop Relat Res. 1994;300:127-32.

2. Allman FL Jr. Fractures and ligamentous injuries of the clavicle and its articulation. J Bone Joint Surg Am. 1967;49:774-84.

3. Robinson CM. Fractures of the clavicle in the adult. Epidemiology and classification. J Bone Joint Surg Br. 1998;80(3):476-84.

4. Lörz M, Bettinger R, Desloovere C, Leppek R. Clavicle fractures after radical neck dissection. HNO. 1991;39(4):147-50.

5. Strauss M, Bushey MJ, Chung C, Baum S. Fracture of the clavicle following radical neck dissection and postoperative radiotherapy: a case report and review of the literature. Laryngoscope. 1982;92(11):1304-7.

6. Harish K. Neck dissections: radical to conservative. World J Surg Oncol. 2005;3:21.

7. Nariai Y, Odawara S, Ichiyama T, Akutsu J, Kanno T, Sekine $\mathrm{J}$. Fracture of the clavicle following radical neck dissection and reconstruction using pectoralis major myocutaneous flap accompanied by postoperative radiotherapy. Craniomaxillofac Trauma Reconstr. 2018;11(2):138-41.

8. Kanda T, Kitajima K, Saito M, Otsuki N, Nibu K, Sugimura K. Insufficiency fracture of the clavicle after neck dissection: imaging features. Jpn J Radiol. 2014;32(03):172-8.

9. Temesvari A, Vandor F. Complications after cervical dissections. (in Gramany) Chirurg. 1954;25:437-43.

10. Ord RA, Langdon JD. Stress fracture of the clavicle. A rare late complication of radical neck dissection. J Maxillofac Surg. 1986;14(05):281-4.

11. Cummings CW, First R. Stress fracture of the clavicle after a radical neck dissection. Case report Plast Reconstr Surg. 1975;55(03):366-7.

12. Pfeifle K, Koch H, Rehrmann A, Nwoku AL. Pseudotumours of the clavicle following neck dissection. J Maxillofac Surg. 1974;2:14-8.

13. Shodo R, Sato Y, Ota H, Horii A. Clavicle fracture with osteomyelitis after neck dissection and post-operative radiotherapy: case report. J Laryngol Otol. 2017;131(11):1026-9.

14. Fujimoto K, Kato H, Kaneko Y, Aoki M, Kuze B, Kato K, Shibata T, Matsuo M. Clavicle fracture following neck dissection: imaging features and natural course. Br J Radiol. 2019;92(1100):20190054.

15. Gorman JB, Stone RT, Keats TE. Changes in the sternoclavicular joint following radical neck dissection. Am J Roentgenol Radium Ther Nucl Med. 1971;111(03):584-7.

16. Soares CBG, Araújo IDD, Pádua BJ, Vilela JCS, Souza RHR, Teixeira LEM. Pathological fracture after radiotherapy: systematic review of literature. Rev da Assoc Méd Br. 1992;65(6):902-8.

17. Lu H, Hu MI, Gagel RF. Cancer therapies and bone health. Curr Rheumatol Rep. 2010;12:177-85.

Publisher's Note Springer Nature remains neutral with regard to jurisdictional claims in published maps and institutional affiliations. 